www.nature.com/hr

\title{
COMMENTARY
}

\section{Is CKD a surrogate marker for predicting cognitive impairment?}

\author{
Masaki Mogi and Masatsugu Horiuchi \\ Hypertension Research (2011) 34, 1251-1252; doi:10.1038/hr.2011.148; published online 1 September 2011
}

$\mathrm{B}$ ecause medical approaches to cognitive impairment (CI) in its progressive state are limited, it is expected that early detection of CI may be the key to preventing worsening of quality of life and reducing the profound disease burden, morbidity and mortality. However, there are few markers for evaluating the future risk of CI in subjects. Recently, impaired kidney function was reported to be associated with dementia and CI. For example, the Reasons for Geographic and Racial Differences in Stroke (REGARDS) Study showed an association between reduced kidney function and CI. ${ }^{1}$ Other studies have also indicated that patients with more severe chronic kidney disease (CKD) have a higher risk of CI. ${ }^{2-4}$ In a recent study in Japan, Yano et al. ${ }^{5}$ found that hypertensives with CKD experience more stroke events compared with hypertensives without $\mathrm{CKD}$, indicating that CKD is an independent predictor of stroke in Japanese patients.

The clinical interaction between the brain and the kidney has been referred to as the 'cerebro-renal connection,' as we previously reported. ${ }^{6}$ Unlike most organs, the kidney and brain are low-resistance end organs that are exposed to high-volume blood flow throughout the cardiac cycle. These hemodynamic similarities are observed in the vascular beds of both the organs. ${ }^{7}$ Ito et al., ${ }^{8}$ in their 'strain vessel hypothesis,' proposed that this similar condition, in which high blood flow leads to disturbances may be a mechanism for cerebro-cardio-renal interactions. Because the small vessels in the brain are similar to those in the kidney, small-vessel disease (SVD) in the kidney may alert us to the presence of

Dr M Mogi and M Horiuchi are at the Department of Molecular Cardiovascular Biology and Pharmacology, Ehime University, Graduate School of Medicine, Tohon, Ehime 791-0295, Japan.

E-mail: mmogi@m.ehime-u.ac.jp
SVD in the brain. Recently, changes in the vascular system in the brain have been shown to contribute to the onset and progression of CI and dementia. ${ }^{9}$ Impairment of the socalled 'neurovascular unit' is linked to many common human CNS pathological conditions, including dementia. Although multiple mechanisms are involved in $\mathrm{CI}$ and dementia associated with $\mathrm{CKD}$, cerebrovascular disease in the brain is considered to have a key role in dysfunction of the neurovascular unit and result in $\mathrm{CI}$ and dementia.

In a study reported in this issue, Yamamoto et al. ${ }^{10}$ clearly demonstrated that brain SVD, such as lacunar infarction and white matter lesions, is associated not only with CI, but also with CKD in Japanese subjects. The authors followed 245 consecutive patients with lacunar infarction assessed by magnetic resonance imaging and ambulatory blood pressure monitoring. They found an independent association between $\mathrm{CKD}$ and $\mathrm{CI}$, which was defined as a Mini-Mental State Examination (MMSE) score of 24 or less. Even moderately reduced kidney function (estimated glomerular filtration rate $(\mathrm{eGFR})=$ 30-60: stage 3) has a higher odds ratio (OR) for mild CI (MCI), which was defined as an MMSE score of 25-27 (OR: 1.77) and CI (OR: 3.69), indicating that patients with eGFR $\leqslant 60 \mathrm{ml} \mathrm{min}^{-1}$ per $1.73 \mathrm{~m}^{2}$ may have CI. The results lead to the important suggestion that it is sometimes advisable for clinicians to administer the MMSE to patients with moderate CKD. However, given the cross-sectional study design (which was similar to that of the REGARDS study), these findings do not prove that CKD actually predicts CI. To explore additional enhancement factors for $\mathrm{CI}$ in CKD patients, and to address the effect of CKD progression, a longitudinal study focusing on the relationship between CI and CKD is needed.
Yamamoto et al. ${ }^{10}$ also showed that not only the mean blood pressure, but also the proportion of dippers and risers as identified via 24 -h ambulatory blood pressure monitoring is higher among patients with SVD, including lacunar infarction and white matter lesions, and CI, indicating that controlling blood pressure throughout the day (known as perfect control of blood pressure) is important to prevent brain SVD and CI.

Ischemic stroke due to SVD is thus far considered to have a better outcome compared with ischemic stroke due to large artery atherosclerosis and cardioembolism. However, in a very recent report, ultra-long follow-up (12 years) of patients with ischemic stroke due to SVD revealed poor long-term survival and an increase in risk of cardiac death compared with other types of stroke, ${ }^{11}$ indicating that patients with this type of stroke may have a high risk of cardiovascular disorders, as well as of cerebrovascular disorders. Common risk factors for SVD, such as high arterial blood pressure and diabetes mellitus, are thought to lead to progression of SVD; therefore, clinicians should provide systematic care to patients who have markers for SVD (in even one organ) to help prevent multiple-organ failure.

\section{CONFLICT OF INTEREST}

The authors declare no conflict of interest.

\section{ACKNOWLEDGEMENTS}

This study was supported by grants from the Ministry of Education, Science, Sports, and Culture of Japan to MH and MM.

1 Kurella Tamura M, Wadley V, Yaffe K, McClure LA Howard G, Go R, Allman RM, Warnock DG, McClellan W. Kidney function and cognitive impairment in US adults: the reasons for geographic and racial differences in stroke (REGARDS) study. Am J Kidney Dis 2008; 52: 227-234. 
2 Yaffe K, Ackerson L, Tamura MK, Le Blanc P, Kusek JW, Sehgal AR, Cohen D, Anderson C, Appel L, Desalvo K, Ojo A, Seliger S, Robinson N, Makos G, Go AS. Chronic kidney disease and cognitive function in older adults: findings from the Chronic Renal Insufficiency Cohort Cognitive study. J Am Geriatr Soc 2010; 58: 338-345.

3 Buchman AS, Tanne D, Boyle PA, Shah RC, Leurgans $\mathrm{SE}$, Bennett DA. Kidney function is associated with the rate of cognitive decline in the elderly. Neurology 2009; 73: 920-927.

4 Jassal SK, Kritz-Silverstein D, Barrett-Connor E. A prospective study of albuminuria and cognitive function in older adults: the Rancho Bernardo study. Am J Epidemiol 2010; 171: 277-286.
5 Yano Y, Hoshide S, Etoh T, Tamaki N, Yokota N, Kario K. Synergistic effect of chronic kidney disease and high circulatory norepinephrine level on stroke risk in Japanese hypertensive patients. Atherosclerosis 2011 (e-pub ahead of print 23 June 2011; doi:10.106/j-atherosclerosis. 2011.06.030)

6 Mogi M, Horiuchi M. Clinical interaction between brain and kidney in small vessel disease. Cardiol Res Pract 2011; 2011: 306189.

7 O'Rourke MF, Safar ME. Relationship between aortic stiffening and microvascular disease in brain and kidney: Cause and logic of therapy. Hypertension 2005; 46: 200-204.

8 Ito S, Nagasawa T, Abe M, Mori T. Strain vessel hypothesis: a viewpoint for linkage of albuminuria and cerebro-cardiovascular risk. Hypertens Res 2009; 32 : 115-121.

9 Zlokovic BV. New therapeutic targets in the neurovascular pathway in Alzheimer's disease. Neurotherapeutics 2008; 5: 409-414.

10 Yamamoto Y, Ohara T, Nagakane Y, Tanaka E, Morii F, Koizumi T, Akiguchi I. Chronic kidney disease, 24-h blood pressure and small vessel diseases are independently associated with cognitive impairment in lacunar infarct patients. Hypertens Res 2011; 34: 1276-1282.

11 Melkas S, Putaala J, Oksala NK, Pohjasvaara T, Oksala A, Kaste M, Karhunen PJ, Erkinjuntti T. Small-vessel disease relates to poor poststroke survival in a 12-year follow-up. Neurology 2011; 76: 734-739. 